\title{
A Real-time Interactive Instructor Framework for Distance Education
}

\begin{abstract}
Xinyou Zhao
Computer Science

Guilin, China

youzx@gliet.edu.cn

\section{Abstract}

Virtual classroom based on Internet is a promising tool for distance education. However, it is difficult for the instructor to instantly know the feedback and evaluation to learners in online courses. The paper gives one instructor-oriented framework for virtual classroom, by which the instructor may know each learner in real time and instantly adjust his approach to teaching. Moreover, in order to improve the participators' freedom for learning \& teaching, electronic board will also be introduced based on PowerPoint technology. These may provide a good face-to-face interaction among participators. At last, problem-based teaching technology introduced improves learning efficiency \& interests.
\end{abstract}

Mitsuji Matsumoto

GITS Social and Information Sciences

Guilin University of Electronic Technology Waseda University Jumonji University

Tokyo, Japan

mmatsumoto@waseda.jp ennmission@gmail.com

\section{Introduction}

When separated with the instructor by space and/or time, learners staying far away can participate in a course live. The system is known as distance education system. Although distance education has many advantages, current systems have not worked well. First, most systems are for learners. The instructors can't see the reflection of learners to his course. So the instructor can't dynamically adjust his class during class. Second, the instructor can only use the slides and can't give his ideas by drawing freely. Third, these virtual classes are passive learning modes.

\section{Distance Learning System Model}

There are four main kinds of multimedia communication technology used in modern distance learning ${ }^{[1]}$ : 1) Audio Technology; 2) Video
Technology; 3) Data Transmission Technology; 4) Print Transmission Technology. During class, it will produce three kinds of videos: instructor's video, learner's video and course's video. Based on these above, we propose one system model of distance education for participators, which contains three exchanging videos based on the source of video: instructor's video, shared resource video, learner's video(Fig.2-1). Under central control, the video of the instructor, course and learner can work well together.

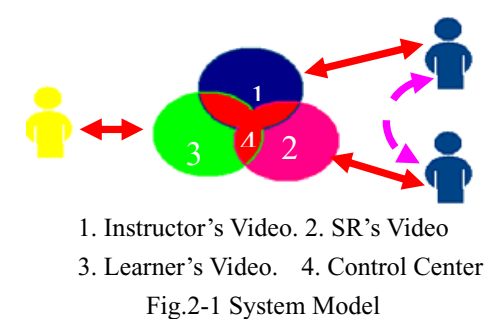

\section{Proposed Framework}

The system framework is shown in Fig3-1.There are two agents for the system, which are instructor agent (IA) and learner agent (LA). The IA and LA maintain connection \& communication among learners and the instructor.

When one learner has successfully loginned in the server, the LA will download the latest contents about course from some learners by $\mathrm{P} 2 \mathrm{P}$ technology in order to reduce the communication burden of the server. Moreover, it will distribute the video and instruction of learners (requests, drawing by mouse etc). Another, LA will connect the video and instruction of instructor some changing slides, drawing traces on e-board, etc . On the contrary, the IA takes two responsibilities: one is the distribution of the video and instruction of instructor, and another is the collection of video and instruction of learners. 


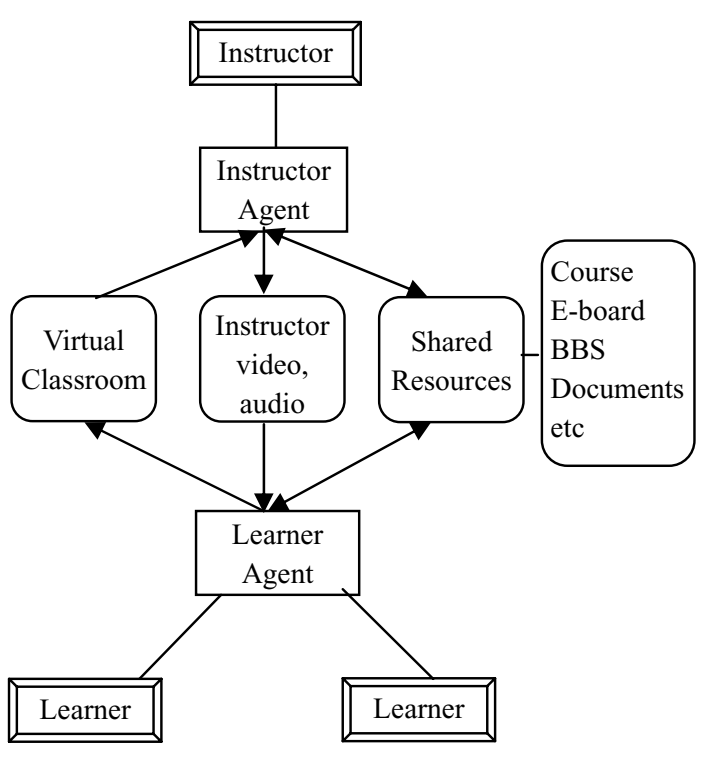

Fig.3-1 System Framework

\subsection{Virtual Classroom}

At traditional classroom, the instructor may see reflection of each learner and know whether the learners have understood the course.

Here, we adopt one virtual classroom model to make the instructor know the reflection of learners. In model, there are many empty sites for learners. When each learner's video is transmitted to the instructor terminal, the system will embed it into one empty desk. Finally, one virtual classroom or virtual group is formed for the instructor. The instructor may see all learners.

\subsection{Problem-Based Teaching -PBT}

The system adopts one synchronous style of problem-based teaching. First, the instructor will group and assign different problems for every virtual group. Then every group will discuss the problem based on problem-based learning $(\mathrm{PBL})^{[2]}$. The instructor may know all groups' discussion and also answer questions of learners. Moreover, every group's discussion will be recorded and be shared with other group after the discussion.

\subsection{Video Separation of Instructor and Course}

As we know, the videos of instructor and the courseware are very important to learners. If they are transmitted to the terminal of the learners together, the system will produce high bps. In our proposed system, we adopt one separated video model for distance system. To instructor's video, we adopt real time transmitting. To courseware, the learner may download from the server before the class. So while the class is going on, we only distribute the instructor video to the learners in real time. When the instructor has changed the contents of courseware, the proposed system will send one instruction to all learners, by whose instruction the terminal of learner may change the content synchronously.

\subsection{Electronic Shared Board System}

In traditional class, the instructor may write out his ideas on one blackboard at will. While the instructor is giving his course by slides, it is difficult to demonstrate some ideas in class, whose ideas are not included in the courseware.

In proposed system, the instructor may point his key knowledge by his hands or one device to one touching screen during his course. After the system recognizes the touching point, it will produce coordinates of touching point and transmit the coordinates to learners. After the learning system receives the coordinates, it will auto-produce one baton pointing the key point according to the size of learners video window.

Another, when the instructor may use the electronic board (by inserting one blank slide), the system only transmits coordinates of drawing trace. Then the learning system will redraw the traces on a blank slide of learner's terminal. Because the system only transmits the coordinates, it needs a low band. Moreover, the learners may use the shared e-board by mouse. It is very cheap for learners to use e-board.

\section{Conclusions}

The paper proposes one virtual classroom framework for the instructor. He/she may not only see all the learners' reflection, but freely use the e-board. These may get one good interaction among participators. Moreover, it improves learning efficiency \& interests for problem-based teaching technology introduced in the system. In future, we will develop the system to provide a virtual classroom for the instructor.

\section{References}

[1] Xinyou Zhao; Yanru Zhong; Mitsuji Matsumoto. A Real-Time Interactive Shared System for Distance Learning The $12^{\text {th }}$ IEEE International Conference on MultiMedia Modelling, Beijing. 2006(1):102-107.

[2] http://www.udel.edu/pbl/ visited on October, 2005 